\section{PF.09 CHROMOSOMAL MICROARRAY (CMA) USE FOR THE PRENATAL DETECTION OF CHROMOSOME ANOMALIES: MODEL-BASED HEALTH ECONOMIC EVALUATION}

doi:10.1136/archdischild-2013-303966.021

${ }^{1} \mathrm{SC}$ Hillman, ${ }^{2} \mathrm{PM}$ Barton, ${ }^{2} \mathrm{TE}$ Roberts, ${ }^{1} \mathrm{ER}$ Maher, ${ }^{1,3} \mathrm{MD}$ Kilby. ${ }^{1}$ School of Clinical and Experimental Medicine, College of Medicine and Dentistry, University of Birmingham, Birmingham, UK; ${ }^{2}$ Health Economics Unit, School of health and populations Science, University of Birmingham, Birmingham, UK; ${ }^{3}$ Fetal Medicine Centre, Birmingham Women's Foundation Trust, Birmingham, UK

Chromosomal microarray (CMA) technology is increasingly used to detect chromosomal anomalies in the prenatal setting. Although its increased detection rates over karyotyping has been proven the cost-effectiveness is yet to be established.

A decision tree was built and populated using two data sets; that of the literature from 21 journal articles and 13755 fetal samples where there was a range of indications for testing and data from 17 cohorts where the indication for testing was abnormalities on fetal ultrasound scan, 4276 fetal samples are included. Costs included were those incurred by the West Midlands Genetics laboratory. Staff costs were taken from Unit costs of health and social care 2011.

When the base case cost of CMA is $£ 405$ and there is a range of indications for performing testing, CMA will cost an extra $£ 6800$ per case detected over karyotyping. CMA will cost an extra $£ 2600$ over karyotyping per case detected when the indication is abnormal ultrasound scan. Threshold analysis shows that when the cost of CMA is reduced to $£ 220$ it becomes the cost-effective option. Cost effectiveness association curves show that for any referral indication when the willingness to pay is $>£ 7500$ and for abnormal scans when the WTP is $>2830$ the probability of CMA being cost effective is over $95 \%$.

CMA will become the cost effective option of the future as technology costs fall. At the present time for abnormal ultrasound scan CMA may be considered if the WTP per case detected is $£ 2600$ in addition to standard costs.

\section{PF.10 THE PLUTO STUDY: EVALUATION OF THE EFFECTIVENESS OF PERCUTANEOUS VESICOAMNIOTIC SHUNTING (VAS) FOR LOWER URINARY TRACT OBSTRUCTION (LUTO), A COMPARISON OF RCT AND REGISTRY DATA}

doi:10.1136/archdischild-2013-303966.022

RK Morris, GL Malin, E Quinlan-Jones, LM Middleton, KS Khan, JJ Deeks, DM Kilby. University of Birmingham, Birmingham, UK

Objective RCT and registry to determine effectiveness of VAS in LUTO for survival and renal function.

Setting Fetal Medicine departments (England, Scotland, Ireland, Netherlands).

Participants Pregnant women with singleton, male fetus with isolated LUTO.

Design RCT with registry. Intervention - VAS compared to conservative care.

Main Outcome Measures Perinatal mortality and serum creatinine at 6 weeks of age and 1 year. Prognostic value of gestational age and liquor volume at diagnosis were assessed in a logistic regression analysis using data from registry and RCT.

Results RCT survival to 28 days favoured VAS: intention-totreat relative risk $1.88,95 \% \mathrm{CI}$ : 0.71 to $4.96(50 \%$ v $27 \%, \mathrm{p}=0.27)$ registry favoured conservative management: relative risk 0.58 , 95\%CI: $0.26,1.29(40 \%$ v $69 \%, p=0.14)$. Those women who entered the registry and had conservative management were more likely to have a normal liquor volume at diagnosis $\left(>5^{\text {th }}\right.$ centile) than those receiving VAS $(p=0.04)$ or those randomised $(p=0.03)$. They also had a higher proportion with gestational age at diagnosis $>24$ weeks compared with those randomised $(p=0.002)$. These variables were strongly associated with improved survival to 28 days in a multivariate logistic regression analysis. There was also a difference in the pathological diagnoses made postnatally in the conservative group in the registry with a $5 / 21(24 \%)$ of babies with a false positive antenatal diagnosis of LUTO.

Conclusion Normal liquor volume $\left(>5^{\text {th }}\right.$ centile) and age at diagnosis $>24$ weeks seem to be associated with increased probability of survival at 28 days in fetuses with confirmed LUTO.

\section{PF.11 ABNORMAL PLATELET FUNCTION IS SEEN IN WOMEN WITH UNEXPLAINED RECURRENT MISCARRIAGE DURING PREGNANCY}

doi:10.1136/archdischild-2013-303966.023

${ }^{1} \mathrm{M}$ Dempsey, ${ }^{1} \mathrm{~K}$ Flood, ${ }^{1} \mathrm{~N}$ Burke, ${ }^{1} \mathrm{~A}$ Murray, ${ }^{1} \mathrm{~S}$ Mullers, ${ }^{2} \mathrm{~L}$ Fay, ${ }^{1} \mathrm{~B}$ Cotter, ${ }^{2} \mathrm{P}$ Fletcher, ${ }^{2} \mathrm{M}$ Geary, 'D Kenny, ${ }^{1} \mathrm{FD}$ Malone. 'Royal College of Surgeons in Ireland, Dublin, Ireland; ${ }^{2}$ Rotunda Hospital, Dublin, Ireland

Objective To evaluate platelet aggregation in patients with a history of recurrent miscarriage (RM) during a subsequent successful pregnancy and compare them to healthy pregnant controls.

Study design A prospective longitudinal study was performed to compare platelet function in 30 patients with a history of three consecutive unexplained first trimester pregnancy losses and 30 healthy age-matched pregnant controls. Exclusion criteria included the use of anti-platelet medications such as aspirin and medical conditions that can affect platelet function. Light transmission aggregometry was used to assay platelet agonists at different times and concentrations to create dose-response curves.

Results In contrast, to the increased platelet aggregation response seen in healthy controls, platelet reactivity in patients with RM peaked at 12-14 weeks gestation, highlighted by the increased aggregation response to epinephrine $(p=0.0008)$ and collagen $(p<0.0001)$ and then decreased in the third trimester in response to epinephrine $(p<0.0001)$, arachidonic acid $(p<0.0001)$ and Thrombin Receptor Activating Peptide $(\mathrm{p}<0.0001)$.

Conclusion Patients with a history of recurrent miscarriage have significantly different platelet function when compared to healthy controls, in particular during the first trimester. Knowledge of which patients have impaired platelet function may allow for more targeted therapy in the setting of recurrent miscarriage.

\section{PF.12 LONGITUDINAL DATA ON FETAL THIGH SOFT-TISSUE PARAMETERS AND ITS ROLE IN THE PREDICTION OF BIRTHWEIGHT}

doi:10.1136/archdischild-2013-303966.024

${ }^{1,2} \mathrm{Cl} \mathrm{O}^{\prime} \mathrm{C}$ onnor, ${ }^{1} \mathrm{~N}$ Farah, ${ }^{1} \mathrm{~A}$ O'Higgins, ${ }^{3} \mathrm{~T}$ Grant, ${ }^{1} \mathrm{C}$ Fitzpatrick, 'B Stuart, ${ }^{1} \mathrm{MJ}$ Turner, 1,2MM Kennelly. 'UCD Centre for Human Reproduction, Coombe Women \& Infants University Hospital, Dublin, Ireland; '2Ultrasound and Fetal Medicine Centre, Coombe Women \& Infants University Hospital, Dublin, Ireland: ${ }^{3}$ CSTAR UCD, Dublin, Ireland

Objective To profile longitudinal changes in the fetal mid thigh muscle and subcutaneous tissue measurements in fetuses with a normal growth velocity in euglycemic pregnancies and evaluate the association between mid thigh parameters and birthweight.

Methods A prospective longitudinal study of 351 singleton fetuses in euglycemic pregnancies. Reliability of fetal abdominal subcutaneous tissue (FAST) measurements and mid thigh indices including thigh muscle (TM) and thigh fat (TF) between two operators was also reviewed.

Results The FAST and fetal mid thigh muscle and fat in AGA fetuses show a significant increase with gestational age. At 28 weeks 